\title{
Ueber Epithelregeneration und sogenannte freie Kernbildung.
} Von

Walther Flemming,

Professor der Anatomie in Kiel.

(Supplement zu: Beiträge zur Kenntniss der Zelle und ihrer Lebenserscheinungen, Th. II, dies. Archiv 1880, Bd. 18, p. 151.)

Indem die neueren Untersuchungen iuber Zelltheilung und Kernvermehrung aus naheliegenden Gründen besonders die epithelialen Gewebe zum Terrain wählten, haben sie, ausser den näheren Kenntnissen über die Theilungsvorgänge selbst, einen Wahrscheinlichkeitsschluss ergeben, der für die allgemeine Gewebelehre nicht unwichtig ist; er lautet: die Regeneration der Epithelien, wie aller Gewebszellen, geschieht durch Zelltheilung in den tiefen Sehichten, mit Kerntheilung unter den allerwege bekannt gewordenen Erscheinungen der Kernmetamorphose (Karyokinese). Es besteht kein Grand, Vorgänge andrer Art - wie z. B. ,freie Zell- oder Kernbildung* - bei der Epithelzellenvermehrung vorauszusetzen.

Diesem Gedanken habe ich schon vor etwa $1 \frac{1}{2}$ Jahren zwar deutlich, aber sehr kurz Ausdruck gegeben ${ }^{1}$ ); denn er kam mir, nach den vielseitigen und massenhaften Befunden von Kerntheilungsfiguren in wachsenden Epithelgeweben, so selbstverständlich vor, dass es mir nicht erforderlich schien ihn noch besonders zu commentiren. In Letzterem habe ich mich getäuscht: eine eben erschienene Arbeit $^{2}$ ) zeigt, wie fest die alten

1) Dies Archiv, Bd. XVI, p. 397.

2) Die physiologische Regeneration des Flimmerepithels der Trachea. Von Dr. Ot to Drasch. Sitzungsber. d. Wiener Acad. d. Wiss., Math. nat. Cl. B. 80,16 . October 1879 . 
Ideen auch gegenüber den neuen Thatsachen noch haften können, und giebt mir deshalb Anlass hier zur Erwägung za stellen, ob sie dazu in diesem Fall wirklich ein Recht haben.

Zwar weiss ich wohl, dass eine grosse Zahl von Biologen hente an dem anfangs hervorgehobenen Satz nicht mehr zweifelt. - Aber auch in neuerer Zeit sind Anschauungen, denen derselbe gegenübertritt, oder mit denen er doch nicht zusammenfällt, von mehreren Seiten geäussert.

Eine so schwerwiegende Stimme wie die Henle's ${ }^{1}$ ) hielt noch vor Kurzem an der Wahrscheinlichkeit fest, dass der Vermehrung der Hautepithelzellen ein freies Entstehen von Kernen nahe der Bindegewebsgrenze, und eine Bildung von Zellen um diese Kerne zu Grunde läge; obschon Henle die Möglichkeit, dass dabei dennoeh Zelltheilungen vorliegen könnten, nicht geradezu ausschliesst.

Dieser Auffassung steht diejenige nahe, wenn auch nicht ganz gleich, welche 1871-1873 Lott ${ }^{2}$ ) durch ansfiuhrliche Arbeiten zu stiutzen suchte; Arbeiten, in welchen zugleich die Formverïnderungen, die die Zellen des Corneaepithels bei ibrem Wachsthum und ibrem Vorrücken gegen die Oberfläche typisch durchmachen, vortrefflich studirt und beschrieben sind. Mit letzterem Theil der Lott'schen Untersuchung habe ich es hier nicht zu thun. In Bezug auf die Lieferungsquelle der neuen Zellen vertritt Lott die Meinung, dass die Fusstheile der nach der Oberfläche aufrückenden Epithelzellen von diesen selbst abgeschnürt werden, und als anfangs kernlose Zellenreste - „Rudimente" Lott - das Keimmaterial für neue Zellen darstellen sollen: dergestalt, dass in diesen Rudimenten Kerne durch Verdichtung sich neubilden, und die Zellen dann nach oben in die Länge wachsen, indem ihre Fusstheile wiederum zurizckbleiben. - Diese Ansicht unterscheidet sich von der vorher angefiuhrten Henle's, wie man sieht, dadurch,

1) J. Henle, Handbuch der Eingeweidelehre. 2. Aufl, p. 3 Anmerkung. Allerdings weiss ich nicht, ob diese Anschauung noch jetzt aufrecht erhalten wird.

2) Ueber den feineren Bau und die physiologische Regeneration der Epithelien, insbesondere der geschichteten Pflasterepithelien. Von Dr. Gustav Lott. Untersuchungen aus dem Institute für Physiologie und Histologie in Graz, herausg. von A. Rollet. 3. Heft, 1873. 
dass sie keine freie Kernzeugung in einem Blastem aufstellt, nähert sich jener aber doch darin, dass sie immerhin eine Kernneubildung ohne Theilung stattfinden lässt, wenn schon im Bereich von Zellprotoplasma.

W. Krause hat dann $1876^{1}$ ) eine Darstellung der Epithelneubildung mit Bezug auf die Cornea gegeben, welche die eben bezeichnete im Wesentlichen wiederholt, ohne Lott's Arbeiten dabei anzuführen ${ }^{2}$ ). - Es ist merkwürdig genug, dass dabei gerade W. Krause selbst, vor all den neueren Arbeiten iber den Gegenstand, die wahren Kerntheilungen im Epithel der Hornhaut gesehen ${ }^{3}$ ), und damit den richtigen Schlüssel zum Verständniss der Epithelregeneration in der Hand gehabt hat, ohne ihn zn benutzen; es liess sich erklärlicher Weise damals noch nicht ahnen, dass diese anscheinend granulirten (eigentlich geknäuelten) Kerne Theilungen seien. Krause hat sie demnach für die Neubildungsfrage ausser Acht gelassen und ist gerade auf das Entgegengesetzte, auf die Lott'sche Hypothese der freien Kernbildung gerathen ${ }^{4}$ ).

Der Glaube an eine solche war so lange vollkommen motivirt, als man noch nichts von den Merkzeichen der Zelltheilung. kannte, welche durch die Kerntheilungserscheinungen geliefert werden. Grade der Umstand, dass sich mit den Untersuchungsmethoden und an den Objecten, die man gerade benutzt hatte, anscheinend keine Kerntheilungen finden lassen wollten - Theilungen, die man noch dazu immer unter dem fälschlich-hergebrachten Bilde einer directen Kernabschnürung suchen zu muissen glaubte,

1) Handbuch der Allgem. Anatomie, 1876, p. 25.

2) Vergl. in der unten besprochenen Arbeit von Drasch, p. I Anm. - Wenn ich selbst (dies. Arch. Bd. 16 p. 397) nicht die Arbeit Lott's, sondern nur die Angabe Krause's kurz erwähnt habe, so geschah dies, weil ich dort nur die Frage nach der Entstehung der Kerne kurz berührt habe, in welcher ich ebensowohl Lott, als seinem Nachfolger Kra use Unrecht geben musste; und weil ich darum glaubte, Lott mit der Erwähnung seiner Priorität in dieser Sache keinen besondern Dienst zu leisten.

3) Allg. und milkr. Anatomie, 1876; Centralbl. f. d. med. Wiss. 1870.

4) Darch eine Mittheilung Pfitzner's bin ich darauf aufmerksam gemacht, dass bereits vor 7 Jahren auch Henle, wie die Fig. 275, 3, 4 in seiner Eingeweidelehre von 1873 zeigt, an Hodenepithelzellen Kerntheilungsfiguren (Knäuelform) mit grosser Treue dargestellt hat, ohne dass sie natürlich damals in ihrer wahren Bedeutung erkannt werden konnten. 
- gerade dieser Umstand ist es ja gewesen, der immer wieder den Gedanken eingab, dass es sich $\mathrm{nm}$ freie Kernbildung handeln müsse.

Da aber eine freie Kernbildung von Niemandem gesehen war und ist, so war dieser Glaube nichts als eine hypothetische Anshuilfe, dieweil man nichts Besseres hatte. Seine Motivirung verschwindet, nachdem zunächst Bütschli, Strasburger u. A. einige der charakteristischsten Formen der Kerntheilung bekannt gemacht, nachdem dann Mayzel und Eberth, Peremeschko und i $\mathrm{ch}^{1}$ ) gezeigt haben, dass diese Kerntheilungsformen in krankhaft- und normalwachsenden Epithelien massenhaft vorkommen, und nachdem endlich Pfitzner und ich ${ }^{2}$ ) gefunden haben, dass diese Theilungen nicht etwa bloss in pathologischen Fällen und bei Larven und Embryen, sondern z. B. anch im geschichteten Hautepithel erwachsener Wirbelthiere constant $z$ u finden sind.

Trotzdem wird jetzt in der vorher angezogenen Arbeit von Drasch wiederum der Versuch gemacht, fuir die Regeneration des Flimmerepithels der Trachea eine freie Kernbildung ${ }^{3}$ ) im Sinne Lott's vorauszusetzen. Die Arbeit ist dabei, was das Studium der Zellenformen und ihrer Wachsthumsveränderungen angeht, so reich an guter Beobachtung und mit so grosser Sorgfalt ausgeführt ${ }^{*}$ ), dass man dadurch bestochen werden könnte, auch

1) Die Literatur s. in diesem Archiv, Bd. XVI, p. 398 und $425 \mathrm{ff}$.

2) S. ebenda, p. 397.

3) Ein für allemal soll bemerkt sein, dass ich den Ausdruck "freie Kernbildung" hier stets im Sinne der bisherigen Ansthauung der Botaniker brauche; wobei das Wort "frei" nicht eine Generatio spontanea in unorganisirtem Blastem bedeutet, sondern nur im Gegensatz zu: „durch Theilung s steht. Ich verstehe also unter „freie Kernbildung: die (hypothetische, unbewiesene) Neuentstehung eines Kerns im Zellprotoplasma dort, wo vorher keiner war; sei es nun wie Lott und Drasch annahmen, durch Verdichtung, oder wie A u erbach wollte, durch Tropfenbildung.

4) Hiervon muss ich uur die Deutung ausnehmen, die Drasch den Becherzellen giebt: er hält sie für vorübergehende Entwicklangsformen der Flimmerepithelzellen. Ich halte dagegen die Ansicht F. E. Schulze's für durchaus richtig, nach der die Becherzellen allerorten, wo sie vorkommen, eigenartige und besonders fungirende Epithelzellen darstellen; und möchte glauben, dass Drasch sich hiervon gleichfalls überzeugt haben würde, wenn er auch andere Epithelarten genauer geprüft, und vor Allem sich auch bei Evertebraten umgesehen hätte. 
die obige Voraussetzung ihres Verfassers als begrindet hinzunehmen. Es scheint mir deshalb richtig, zu zeigen, dass sie dies nicht ist.

Drasch nimmt ebenso wie Lott an, dass ron den aufruckenden Epithelzellen kernlose Rudimente zurückbleiben, in welchen dann neue Kerne durch Verdichtung entstehen, und welche als Ersatzzellen nachwachsen. Für solche freie Kernentstehung bringt Drasch keine neuen positiven Belege, sondern beruft sich (p. 22) auf Lott, bei dem es p. 282 heisst:

„Die so zurïckbleibenden Protoplasmareste (Rudiment-Zellen) bilden nun offenbar die Grundlage zur Bildung neuer kernhaltiger Fusszellen. Diese Kernbildung scheint mir mit einer allgemeinen Verdichtung des Protoplasma zu beginnen, aus dem sich dann der Kern differenzirt. Dafür spricht das Verhalten gegen die Hämatoxylintinction. Man sieht nämlich solche, bei denen sich das Rudiment nur schwach tingirt, während es sich in anderen Fiallen sehr intensiv, fast wie ein Kern tingirt, ohne dass man aber noch einen begrenzten Kern selber wahrnehmen könnte. Ferner spricht dafür die verschiedene Form der Rudimente, indem man ausser den erst beschriebenen (zugespitzte) solche finden kann, deren freies Ende schon wieder vollkommen abgerundet, zuweilen sogar breit ist, ohne dass sie einen Kern zeigen; aber grade diese sind es, die sich stärker tingiren.

Allein auch dies erleidet eine, wenn auch seltnere Ausnahme, indem es vorkommt, dass Kernbildung im untersten Abschnitt schon eintritt, ehe die Trennung vollzogen ist."

Die einzigen Grunde, welche Lott und ebenso Drasch zur Annahme einer freien Kernbildung bestimmt haben, sind also kurzgeordnet folgende:

1. Beide konnten in den tiefsten Epithelschichten und uberhaupt im Epithel keine Kerntheilungen finden.

2. Sie fanden in der tiefsten Schicht kleine, kegelförmige nach oben gespitzte, oder auch anders geformte Protoplasmakörper - "Rudimente" - die sich zuweilen durch nach aufwärts gerichtete Fortsätze mit schon höher gerlickten Zellenleibern in Verbindung zeigten.

3. Sie fanden in den einen dieser "Rudimente" Kerne, in anderen keine solche; und fanden endlich, dass die einen RuArchir f. mikrosk. Anstomie. Bd. 18. 
dimente sich mit Hämatoxylin dunkler färben als die anderen, und als das Protoplasma der höher aufgerïckten Epithelzellen. Sie nahmen solche stärker tingirbare Stellen als Anfänge von Kernneubildung.

1) Der erste dieser Grunde, der negative Befund -beider Forscher in Bezug auf wahre Kerntheilung im modernen Sinn ist nun völlig ohne Bedeutung, angesichts der Methoden und Objecte, die von ihnen benutzt wurden.

Beide Arbeiter nämlich haben vorzugsweise ${ }^{1}$ ) Kali bichromicum oder Müller'sche Lösung zum Isoliren verwendet. Mit der Sorgfalt, die sich wie gesagt im Uebrigen in Drasch's Untersuchung kundgiebt, contrastirt es, dass er sich grade an dies unglucklichste Mittel gehalten hat, das sich zur Ermittlung der Vorgänge an den Kernen nur wählen liess; nachdem ich vor demselben hinlänglich gewarnt zu haben glaubte ${ }^{2}$ ), da ich selbst, ebenso wie Mayzel, mein Lehrgeld durch lange vergebliche Arbeit damit gezahlt hatte.

Da diese dreifach wiederholte Erinnerung von Drasch so ganz ubersehen wurde, erlaube ich mir, zum Frommen ktunftiger Arbeiter, hiermit folgende deutlich sichtbare Warnungstafel aufzustellen:

Wer mit Kali bichromicum oder anderen Chromsalzen Kerntheilangen suchen, oder ansschliessen will, begiebt sich anf einen hoffinungslosen Irrweg.

An den hier citirten Stellen habe ich dies hinlänglich motivirt, indem dort gezeigt wurde, - was auch schon Mayzel erfahren hat - dass die Chromsalze nicht nur die-Structur der

1) Sowie 10 p. c. Kochsalzlösung, die sich, soweit meine Erfahrungen reichen, nicht viel günstiger verbält. - Dass beide Autoren in geringem Maass auch andere Reagentien gebraucht haben, wird unten zur Sprache kommen; dass sie sich im Wesentlichen aber an das Chromkali gehalten haben, geht so deutlich aus ihrer Darstellung, ihren Abbildungen und deren Erklärung hervor, dass ich mit dieser Behauptang kein Unrecht zu begehen glaube.

2) Dies Archiv Bd. XVI, 1878, p. 337 ; Centralblatt f. d. med. Wissenschaften, 1879, 18. Mai, Nr. 23; Virchow's Archiv, Bd. 77, März 1879; p. 19 unten $\mathrm{ff}$. 
ruhenden Kerne stark verändern, sondern noch viel mehr die Kerntheilungsfiguren verstummeln oder zerstören ${ }^{1}$ ).

Ich habe zwar bei fortgesetzten Versuchen mit chromsaurem Kali gefunden, dass man wohl hin und wieder, unter ganz uncontrolirbaren Bedingungen, sehr mässige Erhaltungen von Kerntheilungsfiguren damit erzielen kann; immer sind sie auch dann so undeutlich und verzerrt, dass man schon auf ihren Befund vorbereitet sein, und die unveränderten Formen durch andere Reagentien gut kennen muss, um jene zu diagnosticiren.

Wenn also Lott, Drasch and viele Andere mit dieser Methode keine Kerntheilungen gefunden haben, so ist dies ganz natürlich, beweist aber nicht, dass solche wirklich fehlten.

Beide Forscher haben nun allerdings ansserdem auch Mittel gebraucht, welche Kernfiguren besser erhalten: Chromsäure (L ott p. 268, Drasch p. 2,3), Lott auch Pikrinsäure (p. 270) ${ }^{2}$ ). Aber diese Anwendungen scheinen, nach der Küre, mit der die beiden Autoren ther diese Reagentien hinweggehen, und bei dem Fehlen aller Abbildungen von Präparaten, die damit angefertigt wären, wohl nur kurze und gelegentliche gewesen zu sein.

Ausserdem ist ja bekannt, dass die (bromsäure ${ }^{9}$ ) weit schlechter Epithelzellen isolirt, als die Chromsalze; und ferner ist die Färbung von Chromsäurepräparaten mit Hämatoxylin viel schwieriger und erfordert viel mehr Sorgfalt, als die von Chromkalipräparaten; Drasch und Lott geben auch tiberhaupt nicht an, dass sie Chromsäureobjecte gefärbt hätten. Ohne Tinction aber wird es, bei den kleinzelligen Säugethiergeweben um die es sich hier handelt, sehr schwierig sein und sehr vielen Suchens be-

1) Um nicht missverstanden zu werden, hebe ich wie früher 1. c. hervor, dass diese ungünstigen Eigenschaften der Chromsalze sich im Wesentlichen nur auf die Kerne beziehen, nicht aber auf die Formen von Zellenleibern, welche vielmehr durch sie, wie wohl hinreichend bekannt ist, sehr schön erhalten werden können.

2) Von der von Drasch ebenfalls angewandten Osmiumsäure kann ich absehen, denn sie ist für Kerntheilungspräparate in ihrer Art ebenfalls ganz ungünstig; sie erhält zwar die Theilungen, lässt sie aber so blass, dass sie an kleinkernigen Geweben kaum zu finden sind, und gestattet, wenigstens nach all meiner bisherigen Erfahrung, keine gute Hervorhebung derselben durch Tinction.

3) Pikrinsäure noch viel schlechter. 
düfen, un selbst mit Chromsäure in Isolationspräparaten Theilungen zu finden, es mlissten denn solche gerade in loco in ganz besonderer Masse vorhanden sein.

Ich würde nicht so verfahren, wenn ich im Trachealepithel oder Cornealepithel danach suchen wollte; sondern wirde durch die in Chromsäure oder Pikrinsäure gehärtete Trachea oder Hornhaut in grosser Menge dünne Schnitte legen, diese gut mit Hämatoxylin oder Hermann'scher Anilinfärbung tingiren und aufhellen. Bei diesem Verfahren brauche ich in der Haut einer erwachsenen Salamandra gewöhnlich nur ein halbes Dutzend Schnitte dnrchzusuchen, um eine oder mehrere Kerntheilungen im Epithel zu fiuden; vielfach sind sie auch häufiger. Aber sie sind hier allerdings anch wegen der Grösse viel leichter zu finden. Wollte ich statt dessen das Epithel mit der schlecht macerirenden Chromsüure isoliren, so hätte ich die Wahrscheinlichkeit, auf e in e gut isolirte Zelle mindestens ein Dutzend zu bekommen, die noch zusammenhaften und also die Kerne schlecht erkennen lassen; ich hätte demnach so sehr wenig Chance, Kerntheilungen leicht und klar zu beobachten.

Drasch hat allerdings auch Schnitte angefertigt (mit welchem Reagens, ist nicht gesagt 1. c. p. 3), jedoch, wie er angiebt, nur nm den Situs der Epithelzellen zu überblicken; und ebensowenig bat Lott, nach seinen Worten 1. c. p. 267, das Schnittverfahren cultivirt.

Es geht aus dem Gesagten hervor, dass in dem Trachealepithel und Cornealepithel sehr wohl Kerntheilungen vorkommen können, obschon Lott und Drasch sie bei dem eingeschlagenen Verfahren nicht gefunden haben ${ }^{1}$ ). -

2. Die dem 2 ten Grund (s. o.) entsprechende Thatsache ist völlig zuzugeben; sie hat ja auch mit der Kernneubildung nichts zu thun. Ich stimme Lott und Drasch durchaus darin bei, dass die "Rudimente" zurickgebliebene Theile der aufgerückten Zellkörper sind, die sich von jenen abgeschnürt haben; nur nehme ich dabei an, dass diese Trennung als Zelltheilung und unter Kerntheilung erfolgt, und dass also der eine Tochter-

1) Für Anderes, was dabei noch zur Aufklärung in Rede kommen kann, erlaube ich mir auf den Aufsatz: „Ueber das Verhalten des Kerns bei der Zelltheilung*, Virchow's Archiv 1879, zu verweisen. 
Ueber Epithelregeneration und sogenannte freie Kernbildung.

kern in dem betreffenden "Rudiment" - d. i. der fussständigen Tochterzelle zurückbleibt. - Und damit komme ich auf den dritten Punct.

3. Dass man in Isolationspräparaten aus Chromkali solche "Rudimente" finden kann, die keine Kerne entbalten, in denen man bei guter Tinction die Anwesenheit von solchen sicher ausschliessen kann - dies beweist in der vorliegenden Frage nichts. Denn es sollte jedem geübten Epithel-Isolator bekannt sein, dass man auch bei den besten Einwirkungen, beim schonendsten Arbeiten nicht bloss ganze Zellen isolirt erhält, sondern auch vielfach Zellenbruchstuicke, denen man es oft nicht anmerken kann, ob und wo sie abgebrocben sind.

Dass ferner Zellen der tiefen Epithelschichten sich vielerorten stärker tingïren, als die der mittleren und oberen, ist bekannt. Für ebenso bekannt habe ich es gehalten, dass gerade bei der Hämatoxylinfärbung, auch mit den bestwirkenden Lösungen und bei gleichmässigster Einwirkung in Bezug auf die Kerne ${ }^{1}$ ), das Zellprotoplasma oft recht ungleich im Farbenton ausfällt: nicht nur die eine Zelle dunkler als die andere, sondern selbst ein Theil eines Zellenleibes oft dunkler als der iubrige Theil.

Hiemit muss es sich nun doch von selbst verbieten, dass man aus einer stärkern Tinction eines Protoplasmastiickes schon den weitgehenden und ohne Analogie dastehenden Schluss zielnt, es wolle sich in diesem Protoplasmastück ein Kern bilden.

Aber ich halte es ausserdem für ganz möglich, dass Manches von jenen stärkeren Färbungen in „Rudimenten“, wie sie Lott und Drasch beobachtet haben, wirklieh auf Kernsubstanzen zu beziehen sein mag: nämlich auf Kerntheilungsfiguren ${ }^{2}$ ), die

1) Ich erlaube mir das Obige ziemlich positiv hinzustellen, weil ich auf Grund meiner letzten Arbeiten in Färbungen, und gerade auch Hämatoxylinfärbungen, eine recht ausgedehnte vieljährige Erfahrung habe. Um eine Hämatoxylintinction recht gleichmässig zu erhalten, pflege ich sogar oft die Tinctur um das eingelegte Stück her (durch Schütteln) in fortwährender Bewegung zu halten, und weiss sehr gut, was eine gute, was eine schlechte Färbung der Art ist. Dennoch kann ich versichern, dass auch bei den bestgerathenen Tinctionen, wo alle Kerne im Präparat durchaus die gleiche Nuance haben, in Bezug auf das Zellprotoplasma dabei doch die Ungleichmässigkeiten oft vorkommen, von denen hier oben die Rede ist.

2) In diesen Fällen natürlich Tochterfiguren. Die zugehörige Schwe- 
durch die Chromkalibehandlang verändert waren. Denn diese Veränderungen bestehen zuweilen ${ }^{1}$ ) in einer diffusen $\mathrm{Zu}$ sammenquellung der färbbaren Fadenfigur (Chromatin) mit der nicht färbbaren (Achromatin), mit gleichzeitiger Verwischung der Grenze gegen das Zellplasma; diese Masse färbt sich dann bei Tinction wie ein Kern, aber oft blasser; ein solcher tingirter, schlechtbegrenzter Klumpen kann dann ganz aussehen wie die Dinge, welche Lott und Drasch als Kernneubildungen ansahen.

Wenn man Alles Gesagte berticksichtigt, zeigt sich nicht die mindeste Schwierigkeit für die Annahme, dass die von Lott und Drasch untersuchten Epithelien, wie andere, durch Zelltheilung mit Kerntheilung, unter den allgemeinen Erscheinungen der Karyokinese sich regeneriren. In vollem Einklang steht damit der gewiss richtige Befund beider Forscher, das Vorkommen zweikerniger Zellen in den betreffenden Epithelien betreffend. ${ }^{2}$ ). Wie ich an anderem Orte $^{3}$ ) auseinandergesetzt habe, lassen sich zwei- und mehrkernige Zellen einfach als verunglïckte Zelltheilungen auffassen, bei denen es nur zur Kerntheilung, nicht zur Scheidung des Zellenleibes gekommen ist. Daraus folgt aber auch der Rück-

sterfigur würde in der, von dem betreffenden „Rudiment" abgeschnürten Zelle zu suchen sein.

1) Nicht immer so. In anderen Fällen, und stets dort, wo die Kernmembran bei der Mutter noch erhalten, oder bei den Töchtern schon wieder neugebildet war, erscheint der durch Chromsalze verunstaltete und gefärbte, karyokinetische Kern scharf vom Plasma abgesetzt, im Inneren dagegen entweder gleichmässig tingirt, oder noch mit streifigen verstümmelten Resten der Fadenfigur durchzogen (wie es Taf. XV Fig. $14 \mathrm{~d}$ dies. Archiv, Bd. XVI, andeutet, oft anch in anderen Formen). - Die Sterne und Aequatorialplatten werden aber durch die Chromsalze auch oft in der Art verändert, dass sie sich in Form von unregelmässigen, höckerigen Klumpen im Inneren eines hellen Raums zusammenballen.

Bei den Fällen, um die es sich oben im Text handelt, also bei Tochterkernen in Knäuel- oder Sternform ist noch keine Kernmembran angelegt. kann also jene diffuse Verquellung leicht zu Stande kommen.

$\mathrm{Zu} \mathrm{schwache} \mathrm{Pikrinsäure} \mathrm{verändert} \mathrm{die} \mathrm{Kernfiguren} \mathrm{oft} \mathrm{in} \mathrm{ähnlicher}$ Weise, wie die Chromsalze.

2) Lott p. 275 und a. and. $O$. Drasch scheint in ihrem Antreffen weniger Glück gehabt $z \mathfrak{u}$ haben (p. 12 l. c.), vergl. jedoch seine Fig. IV, 2 und VIII, 3.

3) Virchow's Archiv 1879, Bd. 77: „Ueber das Verhalten des Kerns" etc., p. 15. 
schluss, dass dort, wo man mehrkernige Zellen findet, Kerntheilungen vor sich gehen oder gegangen sind.

Ich habe den Schluss, dass alle Epithelregeneration durch karyokinetische Zelltheilung in den tiefen Schichten vor sich geht, im Eingang dieses Aufsatzes als einen Wahrscheinlichkeitsschluss bezeichnet. Er ist in der That nichts weiter als ein solcher; aber er ist der wahrscheinlichste den wir bis jetzt machen können.

Es lässt sich allerdings nicht ausschliessen, dass entweder noch daneben, oder dass stellenweise sogar allein freie Kernbildung vorkommt. Aber es hat sich eine solche, wie am Schluss noch zu berühren sein wird, bisher nirgends zeigen lassen; während die Neubildung mit Kerntheilung für viele Orte wirklich gezeigt ist. Die Sachlage in dieser Beziehung ist, soviel das Epithel angeht, folgende ${ }^{1}$ ):

Karyokinetische Zelltheilungen sind gefunden: im Epithel der Hautdecke bei Wirbelthierlarven, (Amphibien) Wirbelthierembryen (Vögel, Säugethiere), im Epithel der Hodencanäle (Amphibien, Fische, Arthropoden), im Epithel der Ovarialgänge (Arthropoden, Balbiani), im Epithel der Hornhaut bei erwachsenen Amphibien und Säugethi eren bei Regeneration nach Verletzung, sowie auch (Krause) im normalen Hornhautepithel; endlich im normalen, geschichteten Hautepithel beim erwachsenen Salamander und Triton. Dazu füge ich noch, dass Pfitzner kürzlich auch im Epithel der Darmdrüse $n$ beim erwachsenen Salamander zahlreiche karyokinetische Theilungen fand, von denen mir Präparate vorliegen. -

In vielen dieser Fälle wurden die Theilungen so zahlreich gefunden, dass sie zur Erklärung der gesammten Epithelregeneration völlig ausreichen; in den tibrigen Fällen, wo nur einzelne Theilungen gesehen wurden, war auch die Untersuchung nicht ausgedehnt.

1) Die näheren Angaben, auf die ich für das Folgende verweise, finden sich im I. und II. Theil meiner „Beiträge zur Kenntniss der Zelle und ihrer Lebenserscheinungen", dies Archiv Bd. XVII und XVIII. 
Sollte man nun wirklich, bei dieser Lage der Kenntnisse, annehmen wollen, dass sich z. B. zwar im Hautepithel der Amphibien die Zellen und ihre Kerne durch Theilung fortpflanzen, im Epithel der Trachea aber bei Säugern sich mit freier Kernbildung vermehren? Dass zwar in dem pathologisch wuchernden Hornhautepithel, und unter Umständen auch im normalen, die Kernvermehrung auf dem Wege loyaler Erbfolge durch Theilung geschehe, unter anderen Umständen aber, oder nebenbei, auch durch Generatio spontanea von Kernen im Protoplasma?

Ich kann mir nicht denken, dass ein Biolog, der die Sache ernstlich uberlegt, ohne Notb und AnIass eine Hypothese machen sollte, die eine so gewaltige Ungleichheit einschliesst; dass Jemand dên Werth des Analogieschlusses so gering schätzen sollte, um ihn hier absichtlich nicht anzuwenden. - Desshalb babe ich mir auch nicht die Mühe genommen, im vorliegenden Falle im Epithel der Trachea noch selbst nach Kerntheilungen zu suchen; ich uberlasse es dem, der es will, indem ich mir vorauszusagen erlaube, dass er sie finden wird, wenn er die hier empfohlenen Methoden anwendet. Allerdings würde ich rathen, solche Arbeit zunächst an grosszelligen Geweben, am besten von geschwänzten Amphibien, zu beginnen. -

Ueber das Verhalten der Theilungen im geschichteten Hautepithel habe ich dem kaum etwas hinzuzufiigen, was ich selbst ${ }^{1}$ ) (Salamander) und weiter E. Klein ${ }^{2}$ ) (Triton) bereits dariber ausgesagt haben. Der Annahme des letzteren Autors, dass ausser den indirecten Theilungen noch directe ${ }^{3}$ ) vorkämen, bin ich p. 159 ff. Bd. XVIII dieses Archivs entgegengetreten, inden ich zeigte, dass die indirecten Theilungen für die Erklärung der Regeneration völlig genügen. Näberes äber das Hautepithel wird demnächst Pfitzner mittheilen.

Die Theilungen finden sich hier nicht bloss in der tiefsten Schicht, sondern so weit nach aufwärts, als die Zellen noch nicht eigentlich abgeplattete Formen haben. Es sind das die Schichten

1) Dies Archiv und Virchow's Archiv a. a. 0.

2) Quart. Journ. micr. science, July 1879.

3) Dagegen scheint Klein darin mit mir einig zu sein, dass kein Grund ist hier an eine freie Kernentstehung zu denken. 
die nach Morphologie und Reactionen der Zellen dem Stratum Malpighii der Süugethierhaut entsprechen, welches ja auch bei andern geschichteten Epithelien, als denen der Haut, tiberall sein Aequivalent hat. Um für den langen unbequemen Namen Stratum Malpighii $\left.{ }^{1}\right)^{\circ}$ einen kurzen, und zugleich physiologisch-bezeichnenden zu gewinnen, würde man also deutsch einfach Keimschicht sagen können.

Giebt es ïberbaupt eine freie Kernbildung? Ich habe mir erlaubt, solche hier mehrfach hypothetisch und unbewiesen zu nennen. Dies kann man thun, obne die bezüglichen Angaben vieler und vortrefflicher Beobachter zu ignoriren; und ohne den Forschern selbst damit ein Unrecht zu thun, die aus ihren richtigen Beobachtungen Schlüsse zogen, welche nach der grade herrschenden Meinungsrichtung berechtigt schienen, aber es nicht immer zu bleiben brauchen.

Man erinnere sich nur, wie fest in der Phytologie bis noch vor Kurzem der Glaube an die freie Kernbildung im Embryosack stand, mit welcher Sicherbeit diese Annahme in den Lebrbüchern vorgetragen wurde; und man halte damit zusammen, was Strasburger, der diese Annahme bis dahin selbst getheilt hatte, im vorigen Jahre ${ }^{2}$ ) aussprach:

„Eine freie Kernbildung in den Embryosäcken giebt es nicht, alle Kerne gehen aus einander durch Theilung hervor" -

ein Satz, den Strasburger durch genaue Beschreibung bei vielen Pflanzenformen jetzt hinreichend bewährt. Mit Recht äussert er im Anschluss daran, dass die vorliegenden Angaben über freie Kernbildung im Thierreich einer erneuten Prüfung bedürften. Sollte eine solche nicht vielleicht auch in den sehr einzelnen Fällen aus dem Pflanzenreich ${ }^{3}$ ), für welche Strasburger noch an einer freien

1) Die heute ganz sinnlos gewordenen Namen "Rete Malpighii, Rete mucosum, Stratum mucosum ${ }^{4}$ sind wohl hoffentlich von den meisten Histiologen schon allmählig ausser Curs gesetzt.

2) Botanische Zeitung 1879, 25. April, Nr. 17.

3) Copulation der Spirogyren, Keimung von Ulothrix. Strasburger a. 2. 0. p. 274 . 
Kernbildung festhält, Ergebnisse liefern die mit Anderem mehr in Einklang stehen? - Wir Zoologen haben lang genug gemeint, dass bei der Eitheilung Kerne untergehen und sich neubilden milissten; und haben uns doch geirrt.

Von den vielen Aussagen uber freie Kernbildung bei thierischen Gewebs- oder Eizellen will ich hier nur von den neueren einige der wichtigsten und bekanntesten zur Sprache bringen: zunächst die Angaben von We isman n über die Bildung der Kerne der Polzellen und der Keimhaut bei Insectenembryen 1); die Erfahrungen von Auerbach ${ }^{2}$ ), auf das gleiche Object und auf die Furchung der Froscheier sich beziehend; und die Schilderungen des gleichen Vorgangs vom Rindenprotoplasma des Keims bei

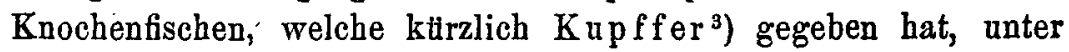
Hinzuziehung der fruheren Angaben E. $\nabla$ on Beneden's, Haeckel's, Lereboullet's, van Bambeke's und Anderer, die sich am letztcitirten Ort zusammengestellt finden.

Man kann die Genauigkeit der betreffenden Beobachtungen so vollkommen anerkennen, wie ich es thue, und doch in ihnen noch keinen sichern Beweis dafür finden, dass in irgend einem dieser Fälle die Kerne durch freie Bildung im Protoplasma, und nicht durch Theilung, entstanden seien. In denjenigen dieser Fälle, welche den Fischkeim betreffen, ist die Furchung desselben bereits vorgeschritten und eine Ableitung der fraglichen Kerne von den schon vorhandenen läge doch wohl im Bereich der Möglichkeit, wenn sie sich auch nicht nachweisen liess. Aber auch wenn man hiervon absieht, und wenn man das Dipterenei heranzieht, bei dem die Erscheinung auftritt, ehe eine anderweite Zelltheilung oder Kerntheilung ersichtlich ist ${ }^{4}$ ): so lässt sich doch heute nicht behaupten, dass der Protoplasmakörper der Eizelle, in dem diese anscheinend freie Kernbildung auftritt, oder die Stellen, an welchen sie auftritt, vorher kernlos waren. In die Substanz dieser Eizelle sind vorher Spermatozoen eingedrungen -

1) Aug. Weismann, Ueber die Entwicklung der Dipteren im Ei. Zeitschr. f. wiss. Zoologie, Bd. 13, 14, 1863.

2) L. Auerbach, Organologische Studien, I, II. p. 82, 85.

3) C. Kupffer, Ueber die Entwicklung des Ostseeherings. Jahresber. der Comm. z. wiss. Unters. der deutschen Meere, Jahrg. 4-6. 1878. p. 200.

4) Vergl. Weismann, a. a. 0. p. 162,169 u. a., Auerbach a. a. 0 . pag. 85 . 
am Wirbelthierei, nach Kupffer's und Hensen's Beobachtungen, in grosser Zahl; - jeder Spermatozoenkopf aequivalent der wirksamen Substanz eines Zellkerns. Wenn sich dieselben bisher nicht morphologisch verfolgen liessen, so ist damit nicht bewiesen, dass sie sich wirklich auflösen und jede Form und Localisation verlieren. Ferner wissen wir nicht, ob an den hier in Rede stehenden Objecten nicht auch der Eikern schon Theilungsproducte abgegeben haben kann; hier, wie dort, ist die bisherige Unmöglichkeit der Verfolgung kein Gegenbeweis. Man braucht sich nur zu erinnern, dass eine Zeit lang auch der Schluss auf den totalen morphologischen Untergang des Eizellenkerns (Keimbläschens) gemacht worden ist, weil sich von ihm za der betreffenden Zeit an ungünstigen Objecten nichts finden lassen wollte - ich rede leider zum Theil aus eigener Erfahrung ${ }^{1)}$ - und dass wir jetzt durch Butschli, O. Hertwig u. A. erfahren haben, wie wenig berechtigt dieser Schluss war.

Es würde mir wenigstens nicht verständlich sein, wenn nach all den neueren Arbeiten uiber die Schicksale des Kerns der Eizelle, und tuber die Richtungskörperbildung, noch Jemand glauben sollte, dass alles dies nur besondere Fälle seien, und dass es wirklich Eizellen gäbe, in denen der ursprlingliche Kern ganz verschwände. Wer diesen Glauben eine Zeit lang theilte, hat um so mehr Anlass auszusprechen, dass derselbe heute nicht mehr berechtigt ist. Denn zu einer solchen Berechtigung würde mindestens ein sicher beobachteter Fall gehören, in welchem der Kern des Eies total untergeht, und einen solchen Fall giebt es nicht; dagegen giebt es ja jetzt zahlreiche, in denen das Gegentheil nachgewiesen ist.

Nicht anders wie in diesen Fällen scheint mir die Sache in denen zu liegen, welche von Seiten der experimentellen Path ologie für eine freie Kernbildung geltend gemacht wurden; wie dies in den bekannten Arbeiten von $\mathrm{Arnold}^{2}$ ) und Klebs ${ }^{3}$ ), auch durch Mayze ${ }^{4}$ ) geschah. Wenn es auch hier überall sehr danach

1) S. dies. Archiv Bd. 16, p. $411-413$.

2) Die Vorgänge bei der Regeneration epithelialer Gebilde. Virchow's Archiv, Bd. 46, 1869, p. 168.

3) Die Regeneration des Plattenepithels. Arch. f. experim. Path. und Pharm. Bd. III.

4) Siehe: Centralbl. f. d. med. Wiss. 1875, Nr. 50 (am/ Schluss). Nach 
aussah, dass die betreffenden Kerne neu und nnabhängig von den praeexistirenden entstehen, und wenn demnach die Untersucher diesen Schluss gezogen haben, so darf man doch sagen, dass ein eigentlicher Beweis für denselben nicht vorliegt. Eberth ${ }^{1}$ ) und Hoffmann ${ }^{2}$ ) sind ihm bereits mit triftigen Gründen entgegengetreten, ohne dass damit andrerseits eine directe Widerlegung geliefert wäre; Eberth selbst spricht sich in dieser Hinsicht sehr vorsichtig, und sehr viel Spielraum lassend aus ${ }^{3}$ ).

Zur Zeit dieser Untersuchungen wusste man eben noch nichts von den Kerntheilungsfiguren ${ }^{4}$ ), von ihrer oftmaligen Blässe und selbst Unsichtbarkeit an lebenden Geweben, von ibrem oft schubweisen Auftreten und ibrem Fehlen in den Intervallen solcher Schitbe ${ }^{5}$ ), endlich von ihren Beeinflussungen durch Reagentien.

In der Haut der Batrachierlarven z. B. finde ich die Theilungsfiguren lebend sehr blass, ohne Reagentien kaum studirbar; es ist deshalb ganz natuirlich, dass die Beobachter der Substanzrerlust-Regeneration, die sich hier an das lebende Object hielten, von diesen Dingen nichts gesehen haben. - Während die ruhenden Kerne des lebenden Hautepithels an der Salamanderlarve deutlich sichtbar sind (dies Arch. Bd. 16 p. 313,361 ff.), erscheinen sie am Kiemenblattepithel derselben, sowie auf der Haut der Tritonlarve, unsichtbar (s. ebenda, und bei Peremeschko, Centralbl. f. d. m. W. 27 Juli 1878); die Theilungsfiguren sind am lebenden

brieflichen Mittheilungen darf ich jedoch vielleicht annehmen, dass auch Mayzel jetzt, nach weiterer Verfolgung der Kerntheilungsvorgänge, die freie Kernbildung in den betreffenden Fällen nicht mehr für so zweifellos hält.

1) Die Regeneration des Hornhautepithels. Virchow's Archiv Bd. 51, 1870, p. 361.

2) Epithelneubildung auf der Cornea. Virchow's Arch. Bd. 51, 1870, pag. 373.

3) Dass wir eine freie Kernbildung neben einfacher und doppelter Theilung der ursprünglichen Kerne für sehr wahrscheinlich halten müssen, womit wir aber die Abkunft der kleinen Kerne von den grösseren nicht läugnen wollen", p. 367 .

4) Mayzel allerdings hatte solche gefunden und in demselben Aufsatz, der oben citirt ist, beschrieben; aber es liess sich damals noch nichts über ihre allgemeine Verbreitung und typische Bedeutung bei der Zelltheilung ahnen.

5) S. den Aufsatz in Virchow's Archiv Bd. 77: Ueber das Verhalten des Kerns bei der Zelltheilung, p. 18. 
Kiemenblatt sehr blass; am Hantepithel deutlicher, aber hier immer noch weit blasser als die ruhenden Kerne; während letzteres sich bei Triton nach Peremeschko gerade umgekehrt verhält. Bei Säugethieren (Kätzchen, Omentum) fand ich die lebenden Kerntheilungen blass bis fast zur Unsichtbarkeit, während hier die ruhenden Kerne auch im Leben ganz deutlich sind.

Bei diesen so grossen Verschiedenheiten der Objecte bedarf es für die Entscheidung der hier besprochenen Frage einer sehr sorgfältigen Auswahl und Vergleichung der letzteren, unter gleichzeitiger Anwendung der massgebenden Reagentien. Das Vermissen von Kerntheilungsfiguren an irgend einem bestimmten lebenden Gewebe giebt, nach dem eben Gesagten, keineswegs eine Gewähr daftir, dass solche wirklich fehlen, und dass sie nicht durch geeignete Zuziehung von Pikrinsäure, Chromsäure, selbst schon Essigsäure, auch hier dargestellt werden könnten, - neben den Dingen und vielleicht sogar zum Theil als die Dinge selbst, welche den Untersuchern der Substanzverlustränder als Zeichen einer freien Kernbildung erschienen sind.

Der Leser dieses Aufsatzes mag den Eindruck bekommen haben, dass ich darin für den Satz: „omnis nucleus e nucleo" eingetreten bin. Ich thue dies aber nicht anders als unter Anhängung der Clausel: so viel wir bis jetzt wissen. Ich zweifle nicht an der Möglichkeit einer freien Zellbildung ${ }^{1}$ ), einer freien Kernbildung, einer Generatio spontanea überhaupt; ich kann die Vorstellung einer solchen nicht einmal, wie Andere es thun, abenteuerlich finden; es scheint mir selbst die Hoffnung, dass sich die Bedingungen für solche Vorgänge einst werden näher erkennen and ktinstlich nachahmen lassen, auf kein blosses Ideal gerichtet.

1) Das, was man jetzt "Zellbildung" oder „freie Zellbildung" zu nennen pflegt - so die betreffenden Vorgänge im Embryosack der Pflanzen (vergl. Strasburger a. a. 0.) und im Rindenplasma von Eiern (Weismann, Kupffer a. a. O.) - ist ja nichts Anderes als eine Territorienscheidung in einem gegebenen lebenden Protoplasmakörper, der mehrere Kerne enthält, also im Grunde nur eine besondere Form der Zelltheilung. Mit dem Obenstehenden aber meine ich auch eine eigentliche Generatio aequivoca von Protoplasma. 
Will man sich aber solchem Ziel nähern, so kann es dazn nicht der geeignete Weg sein, dass man das $x$, das noch gesucht werden soll, die freie Kernbildung, als gegeben und selbstverständlich annimmt ùnd unbedenklich damit rechnet. Als ein viel besserer Weg erscheint es, vor Allem aufs Genaueste und mit immer neuen Mitteln die vitalen Vorgänge der Zellen- und Kernneubildung weiter zu erforschen, wie sie uns die Natur selbst vormacht. Bis jetzt hat diese Forschung mit Sicherheit noch keine andere Art solcher Neubildung gezeigt, als: Zellen fortpflanzung durch Zelltheilung, mit Kernvermehrung durch metamorphotische Kerntheilung.

Kiel, den 17. April 1880.

\title{
Neve Untersuchungen zur Anatomie der Seiten- organe der Fische.
}

\section{Die Seitenorgane der Knochenflsche 1).}

\author{
Von \\ B. Solger \\ in Halle an der Saale.
}

Hierzu Tafel XVII.

Die Ueberschrift dieses dritten Abschnitts, welcher zunächst die Darlegung des thatsächlichen Materials beschliessen, und alsdann das Facit der gesammten Untersuchung ziehen soll, nennt eine Abtheilung der Fische, die der Teleostier, welche in zahlreichen Formen und Individuen die Gewässer unserer Heimath bevölkert. Zu jeder Jahreszeit leicht und reichlich zu beschaffen,

1) I. u. II. s. Bd. XVII dies. Arch., Seite 95 und 458 ff. S. 467, Z. 14 v. o. sind die Worte: ${ }_{n}$ in dorso-ventraler Richtung ${ }^{2}$ zu streichen. 